\title{
Lesions of the Fornix but Not the Entorhinal or Perirhinal Cortex Interfere with Contextual Fear Conditioning
}

\author{
Russell G. Phillips and Joseph E. LeDoux \\ Department of Psychology and Center for Neural Science, New York University, New York, New York 10003
}

The effects of entorhinal cortex lesions, combined entorhinal and perirhinal cortex lesions, and fornix lesions on the conditioning of fear responses (freezing) to contextual stimuli were examined using a conditioning procedure known to produce hlppocampal-dependent contextual conditioning. Lesions of the entorhinal and or entorhinal plus perirhinal cortex did not disrupt contextual conditioning, but lesions of the fornix did. None of the lesions affected conditioning to an explicit conditioned stimulus. Given that the entorhinal cortex is the primary linkage between the neocortex and the hippocampus and that the fornix is the primary linkage with subcortical structures, subcortical inputs to and outputs of the hippocampus appear to be sufficient to mediate contextual fear conditioning. As a result, the presumption that neocortical information is required for contextual fear conditioning, and perhaps other hippocampal-dependent functions, should be reevaluated.

[Key words: context, fear conditioning, memory, hippocampus, entorhinal cortex, fornix]

Recent studies have implicated the hippocampal formation as an essential component of the circuitry through which contextual stimuli acquire aversive properties through fear conditioning (Selden et al., 1991; Kim and Fanselow, 1992, 1993; Phillips and LeDoux, 1992, 1994). It is generally assumed that hippocampal-dependent functions involve the prior processing of sensory information in cortical areas, relay to multimodal processing regions in the parahippocampal region, transmission to the entorhinal cortex, and finally, entry into hippocampal circuitry (Van Hoesen et al., 1972; Swanson and Kohler, 1986; Deacon et al., 1988; Amaral and Witter, 1989). By this logic, damage to the entorhinal cortex should interfere with performance on hippocampal dependent tasks, as the entorhinal cortex is a critical link between the neocortex and the hippocampus, both providing the hippocampus with neocortical inputs and providing the neocortex with hippocampal outputs. Damage to the entorhinal cortex has, in fact, been found to interfere with performance on a number of hippocampal-dependent tasks (Murray et al., 1989; Zola-Morgan et al., 1989; Bunsey and Eichenbaum, 1993; Mumby and Pinel, 1994; Nagahara et al., 1995), but the effects of

\footnotetext{
Received Dec. 16, 1994; revised Mar. 2, 1995; accepted Mar. 6, 1995.

This work was supported by NSF9209646, MH38774, and MH00956; the present experiments were performed in partial fulfillment of Ph.D. by R.G.P. in the Department of Psychology at New York University, 1994.

Correspondence should be addressed to Dr. Joseph LeDoux, Center for Neural Science, New York University, 4 Washington Place, Room 809, New York, NY 10003.

Copyright $(\mathcal{C} 1995$ Society for Neuroscience $0270-6474 / 95 / 155308-08 \$ 05.00 / 0$
}

entorhinal cortex lesions have not been examined in contextual fear conditioning.

In the present study we therefore examined the effects of lesions of entorhinal cortex on contextual fear conditioning. Our failure to find an effect prompted us to perform three additional studies. First, we examined the effect of combined lesions of the entorhinal cortex and perirhinal cortex, since the latter is also known to receive inputs from cortical sensory processing areas and project directly to the hippocampal formation and the amygdala (Deacon èt al., 1983; Turner and Zimmer, 1984; McDonald and Jackson, 1987; Burwell and Amaral, 1993; Romanski and LeDoux, 1993). Connections with the amygdala are of interest since it is an essential component in the fear conditioning circuitry (Davis, 1992; Kapp, 1992; LeDoux, 1992, 1995). Second, we examined the effects of damage to the fornix, which constitutes another main source of input and output connections of the hippocampus (Swanson and Cowan, 1977; Amaral, 1987; Amaral and Witter, 1989). Third, in order to verify that our entorhinal cortex lesions interfered with hippocampal functions that are dependent on the entorhinal cortex, animals with entorhinal cortex lesions were tested on a passive avoidance task, known to be disrupted by manipulations of either the entorhinal cortex (Izquierdo et al., 1993) or the hippocampus (O'Keefe and Nadel, 1978; Gray, 1982).

\section{Materials and Method}

Animals and surgery. Male Sprague-Dawley rats (Hilltop labs), which weighed $250-275 \mathrm{gm}$ upon arrival, were housed in pairs in clear plastic cages. They were provided with free access to lab chow and water and maintained on a $12 \mathrm{hr}: 12 \mathrm{hr}$ light-dark cycle.

About $7 \mathrm{~d}$ after arrival, animals in the entorhinal cortex lesion group (Ent, $n=8)$ were anesthetized with pentobarbital $(40 \mathrm{mg} / \mathrm{kg})$ and mounted in a custom made head holding device. The cranium was exposed and a craniotomy was performed over the posterior cortex. Entorhinal cortex lesions were made by visualizing the rhinal sinus, retracting the dura and slowly aspirating the exposed cortex using a 32 gauge needle attached to a vacuum pump. The extent of the aspiration was guided using superficial landmarks (e.g., bottom of the skull and one millimeter anterior to the crista supratympanica). Hemorrhaging was controlled by application of ice-cold saline and gel foam. For controls $(n=8)$, the craniotomy was performed but the dura was left intact. The same procedures were performed on animals in the combined en torhinal/perirhinal cortex lesion group ( $\operatorname{Ent} \operatorname{Pr}, n=5$ ), but in addition, aspiration lesions extended $2 \mathrm{~mm}$ forward from the crista supratympanica and the medial entorhinal cortex was lesioned electrolytically $(1 \mathrm{~mA}$, anodal direct current, 7-10 sec) using monopolar stainless steel electrodes insulated with epoxy to within $200 \mu \mathrm{m}$ of the tip. Electrode placement was guided by coordinates modified from an atlas of the rat brain (Paxinos and Watson, 1986). The anterior-posterior (AP), mediallateral (ML), and dorsal-ventral (DV) coordinates were computed in relation to the interaural line $(\mathrm{AP}=2.0, \mathrm{ML}= \pm 4.0, \mathrm{DV}=2.0)$. For EntPr controls, only the craniotomy was performed.

For animals in the fornix lesion group ( $\mathrm{FX}, n=8$ ), the fornix and fimbria were lesioned electrolytically. Two lesions were placed in each 
hemisphere $(\mathrm{AP}=7.6, \mathrm{ML}= \pm 0.5, \mathrm{DV}=3.2$ from dura; $\mathrm{AP}=7.6$, $\mathrm{ML}= \pm 1.5, \mathrm{DV}=3.2$ from dura). For controls $(n=6)$ the electrode was lowered to the same locations but no current was passed. After surgery the wound was sutured and the animal was placed under a heat lamp until recovery from anesthesia was complete. Animals were then returned to the housing area and allowed to recover for $14 \mathrm{~d}$ before training began.

Conditioning apparatus and procedure for fear conditioning. Rats were placed individually into a rodent conditioning chamber (Coulbourn Instruments Inc., Lehigh Valley, PA, model E10-10) enclosed by a sound-attenuating cubicle (Coulbourn Instruments Inc., model E10-20). Stimulus presentation was controlled by a microprocessor and a digital I/O board (Opto 22). The CS was a $10 \mathrm{kHz}$ tone produced by a frequency generator (Coulbourn Instruments Inc., model S81-06), amplified to $75 \mathrm{~dB}$, and presented for $20 \mathrm{sec}$ through a speaker located in the front panel of the chamber. The US was a brief $(500 \mathrm{msec})$ distributed delivery of current $(0.5 \mathrm{~mA})$ produced by a grid floor shocker (Coulbourn Instruments Inc., model E13-08)

On day 0 , animals were placed in the conditioning apparatus for 20 min with no stimuli presented. On days 1 and 2, Pavlovian conditioning trials were given during which two CS presentations each coterminated with the US (ITI $=60-120 \mathrm{sec}$ ). On day 3 , control and lesioned groups received the CS on the same schedule as on the previous $2 \mathrm{~d}$, but without US presentation.

Freezing was used as an index of conditioned fear (Blanchard and Blanchard, 1969; Bouton and Bolles, 1980; Fanselow, 1980; LeDoux et al., 1984) and was assessed by viewing the animals through a peephole in the sound-attenuating chamber. Freezing involved the absence of all movement, except for respiratory-related movements, while the animal was in the stereotyped crouching posture (Blanchard and Blanchard, 1969); standing motionless but without crouching was not scored as freezing. Fear conditioning to the phasic CS was measured during the first $20 \mathrm{sec} C S$ presentation on each day. Contextual fear conditioning was assessed by measuring freezing during the $20 \mathrm{sec}$ prior to the onset of the first CS of each day, as previously described (Phillips and LeDoux, 1992, 1994).

Apparatus and procedure for passive avoidance. Several days after extinction criterion was met in the fear conditioning procedure (less than $5 \mathrm{sec}$ of freezing during a trial on 2 consecutive days of extinction trials), Ent animals were placed in a novel environment on a platform $(7 \mathrm{~cm} \times 10 \mathrm{~cm} \times 2 \mathrm{~cm})$ siluated on a grid foor connected to a grid floor shocker (Coulbourn Instruments, model E13-08) in a clear plastic box. Latency to step off the platform was recorded. Two hours later, animals were again placed on the platform and were given a $1 \mathrm{~mA}, 500$ msec footshock when two paws touched the grid floor. On the next day (testing), animals were placed on the platform and the latency to step down was recorded. The procedure was based on Izquierdo et al. (1993).

Histology. After completion of behavioral studies animals were given an overdose of sodium pentobarbital $(120 \mathrm{mg} / \mathrm{kg})$ and transcardially perfused with physiological saline, followed by $10 \%$ buffered formalin Brains were postfixed in buffered formalin for $1 \mathrm{~d}$ to 2 weeks, frozen, and cut coronally on a sliding microtome or cryostat into $40 \mu \mathrm{m} \mathrm{sec}$ tions. Every fourth section was mounted on a gelatin-coated slide, allowed to air dry, and stained with thioneine.

\section{Results}

Entorhinal cortex lesions and fear conditioning

Histological analysis of animals with lesions aimed at the entorhinal cortex revealed that in most cases the vast majority of the entorhinal cortex as well as posterior portions of the perirhinal cortex, and in some cases ventral subiculum and CA1, were removed (see Fig. 1). Animals were excluded from the statistical analysis if there was less than $75 \%$ damage to the entorhinal cortex (extent of damage was estimated from serial reconstruction of coronal sections). Ent animals included in the analysis $(n=8)$ had between $75 \%$ to $100 \%$ of the entorhinal cortex removed (control $n=8$ ).

The effects of entorhinal cortex lesions on the acquisition of freezing responses to contextual cues and to the primary CS were analyzed across days by performing an analysis of variance with one grouping variable [surgery (lesion or control)], and two repeated measures [stimulus type (context or CS) and day (1-

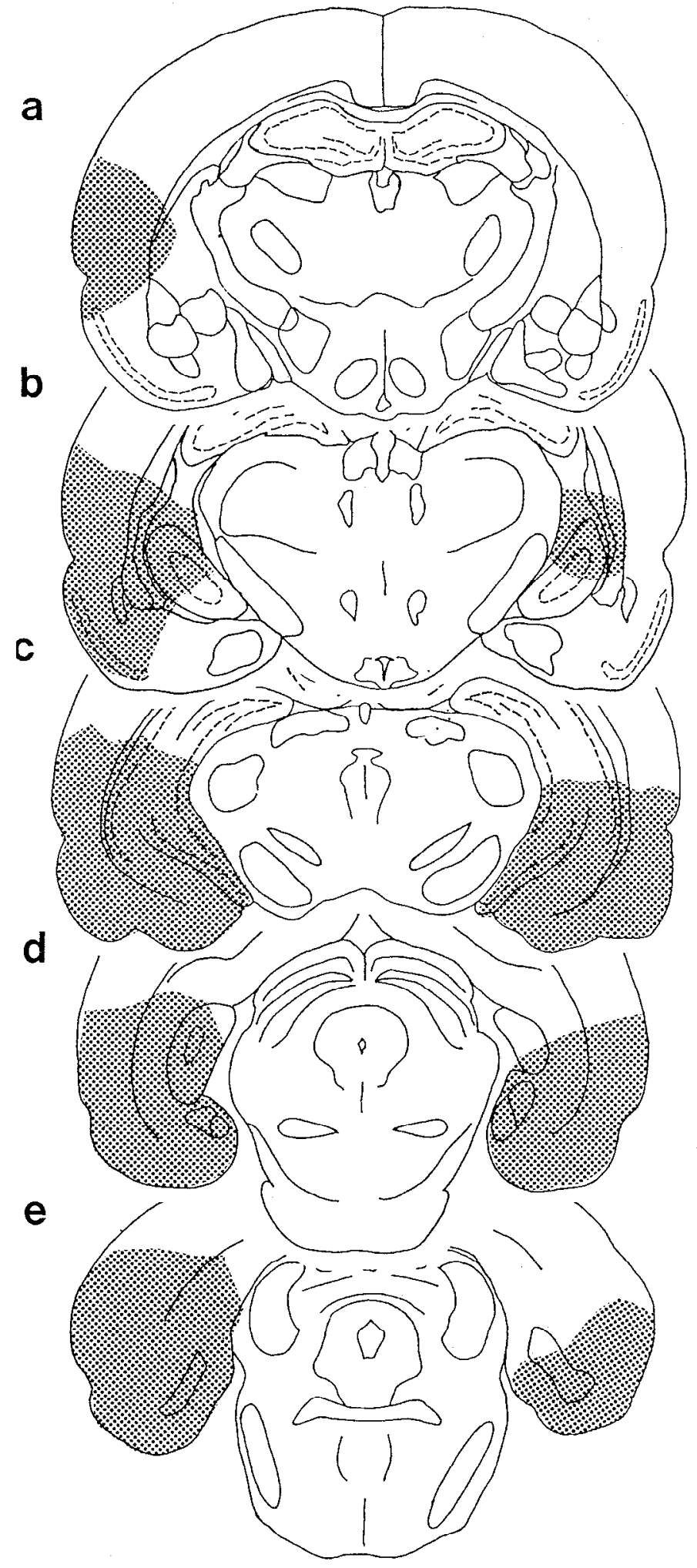

Figure 1. Serial frontal sections illustrating a typical lesion of the entorhinal cortex. Sections are from rostral to caudal $(a-e)$, with stippling indicating the lesion site.

3)]. The main effect of surgery was not significant $[F(1,13)=$ $0.28, p>0.60]$, nor was the interaction between surgery and type $[F(1,13)=2.11, p>0.16]$ or surgery and day $[F(2,26)=$ $0.54, p>0.58]$ or surgery, type, and day $[F(2,26)=0.78, p>$ $0.46]$. The main effect of day $[F(2,26)=71.10, p<0.001]$ and 

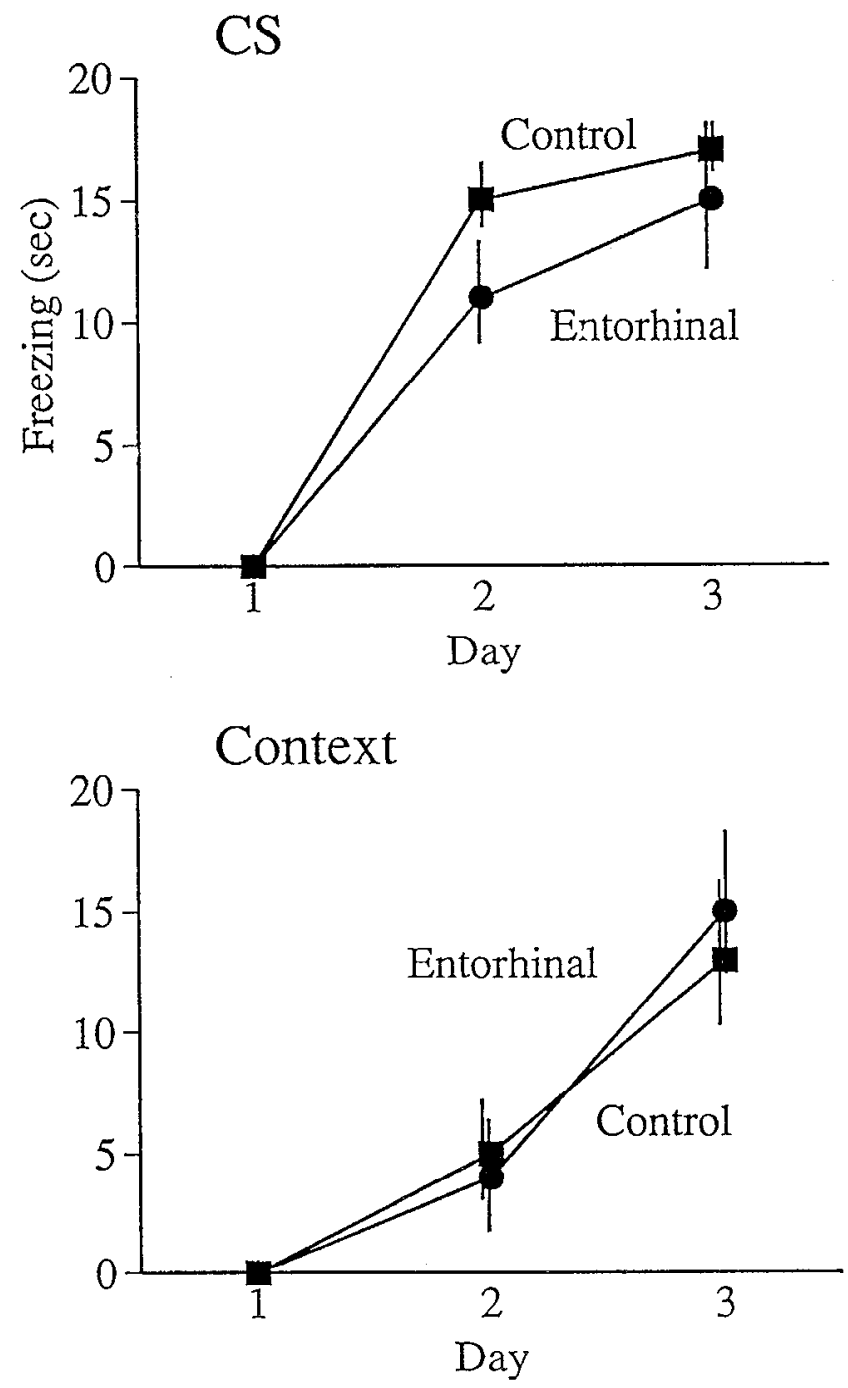

Figure 2. Effects of lesions of entorhinal cortex on the acquisition of conditioned freezing to the conditioned stimulus ( $C S$; a tone) and to the background context. Error bars represent the SE below and above the mean (entorhinal, $n=8$; control, $n=8$ ).

of type $[F(1,13)=17.34, p<0.002]$ were significant. In addition, there was a significant interaction of day and type $[F(2,26)=14.58, p<0.001]$. Thus, there was a difference in acquisition rate between context and CS conditioning in controls, as previously reported (Phillips and LeDoux, 1992), and damage to entorhinal cortex did not affect this (see Fig. 2).

\section{Ent lesions and passive avoidance}

The failure of our Ent lesions to affect hippocampal dependent contextual conditioning led us to examine whether our Ent lesions would affect performance on a step-down passive avoidance task which has been shown to be sensitive to disruption of entorhinal cortex (Izquierdo et al., 1994) and hippocampal function (see O'Keefe and Nadel, 1978; Gray, 1982). The latency to step down from the platform on day 1 (training) was measured in comparison to the latency on day 2 (testing) (Table 1). The effects of entorhinal cortex lesions on this task were analyzed by performing an analysis of variance with one grouping variable [lesion (control or lesion)] and one repeated measure [day (training or testing)]. The main effect of day was significant $[F(1,14)=15.47, p<0.005]$, as well as the interaction between
Table 1. Group averages of step-down latency

\begin{tabular}{llrl} 
Group & $N$ & Training latency & Test latency \\
\hline Sham lesion & 5 & $6.6( \pm 3.1) \mathrm{sec}$ & $93.6( \pm 21.4) \mathrm{sec}^{*}$ \\
Ent lesion & 9 & $17.7(+8.7) \mathrm{sec}$ & $37.0( \pm 19.7) \mathrm{sec}$
\end{tabular}

Data presented are \pm SE, measured in seconds. See Results for further explanation.

$* p<0.001$ compared to controls.

day and lesion $[F(1,14)=6.25, p<0.05]$. Post hoc $t$ tests revealed a significant difference between training and testing for control animals $[t(14)=7.70, p<0.01]$, but not for Ent animals $[t(14)=1.72, p>0.05]$ (see Table 1). Little or no freezing, defined as motionless "crouching," was exhibited by either the control or lesion animals when placed on the platform in the conditioning chamber during training or testing.

\section{EntPr lesions and fear conditioning}

Perirhinal cortex receives inputs from a wide range of cortical areas (Deacon et al., 1983; Romanski and LeDoux, 1994) and projects directly to the hippocampal formation (Kosel et al., 1983). To test the possibility that perirhinal cortex might be an alternative route between cortical areas and the hippocampus that might mediate contextual fear conditioning, we examined the effects of combined lesions of the entorhinal and perirhinal cortex (EntPr). The lesions included much of the medial and lateral entorhinal cortex and the perirhinal cortex as far anterior as the amygdala. Animals were included if more than $75 \%$ of the entorhinal and perirhinal cortex was ablated, with lesion size evaluated from serial reconstructions ( $n=5$; controls, $n=8$ ). A typical lesion is reconstructed in Figure 3. Some lesions included ventral CAl and subiculum, as well as the entorhinal and perirhinal areas.

The effects of EntPr lesions were analyzed using the same design as described above for Ent animals and fear conditioning (ANOVA with one grouping variable and two repeated measures). There was a main effect of day $[F(2,16)=84.0, p<$ $0.001]$, but not surgery, nor an interaction between the two. A main effect of type was significant $[F(1,8)=7.72, p<0.025]$, as well as an interaction of day and type $[F(2,16)=8.51, p<$ 0.025]. No other main effect or interaction was significant (see Fig. 4). There was a difference in the acquisition rate for animals depending on stimulus type, and damage to the rhinal cortex did not affect this.

\section{Fornix lesions and fear conditioning}

The failure of Ent or combined EntPr lesions to affect contextual fear conditioning led us to examine the effects of lesions of the fornix, which provides another major source of hippocampal input and output connections (Swanson and Cowan, 1977; Amaral, 1987). Lesions of the fornix (FX) typically included the fornix as well as the fimbria and in some cases the anterior pole of the hippocampus or part of the medial septum ( $n=8$; see Fig. 5; control $n=6$ ).

The effects of fornix lesions were analyzed using the same design as above. The main effect of day $[F(2,26)=77.26, p<$ $0.001]$ and type $[F(1,13)=10.14, p<0.01]$ and the interaction between day and type $[F(2,26)=5.92, p<0.01]$ were significant. Although the main effect of surgery was not significant, nor the interaction between surgery and day, the interaction of surgery and type was significant $[F(1,13)=4.74, p<0.05]$. In 


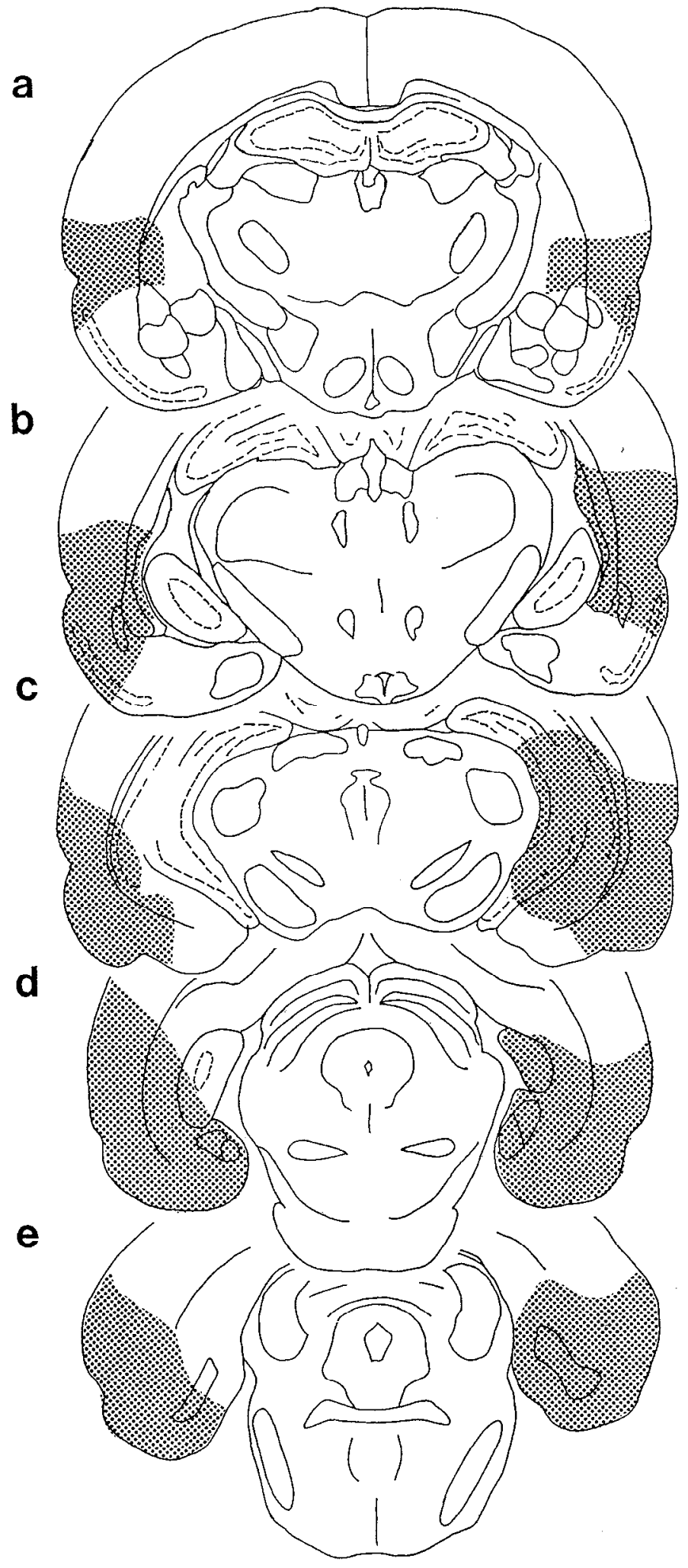

Figure 3. Serial frontal sections illustrating a typical combined lesion of the entorhinal and perirhinal cortex. Sections are from rostral to caudal $(a-e)$, with stippling indicating the lesion site.

addition, the three-way interaction of day, type, and surgery was significant $[F(2,26)=3.40, p<0.05]$, indicating that there was a significant difference between FX animals and their controls, dependent upon the day and on the type of stimulus information
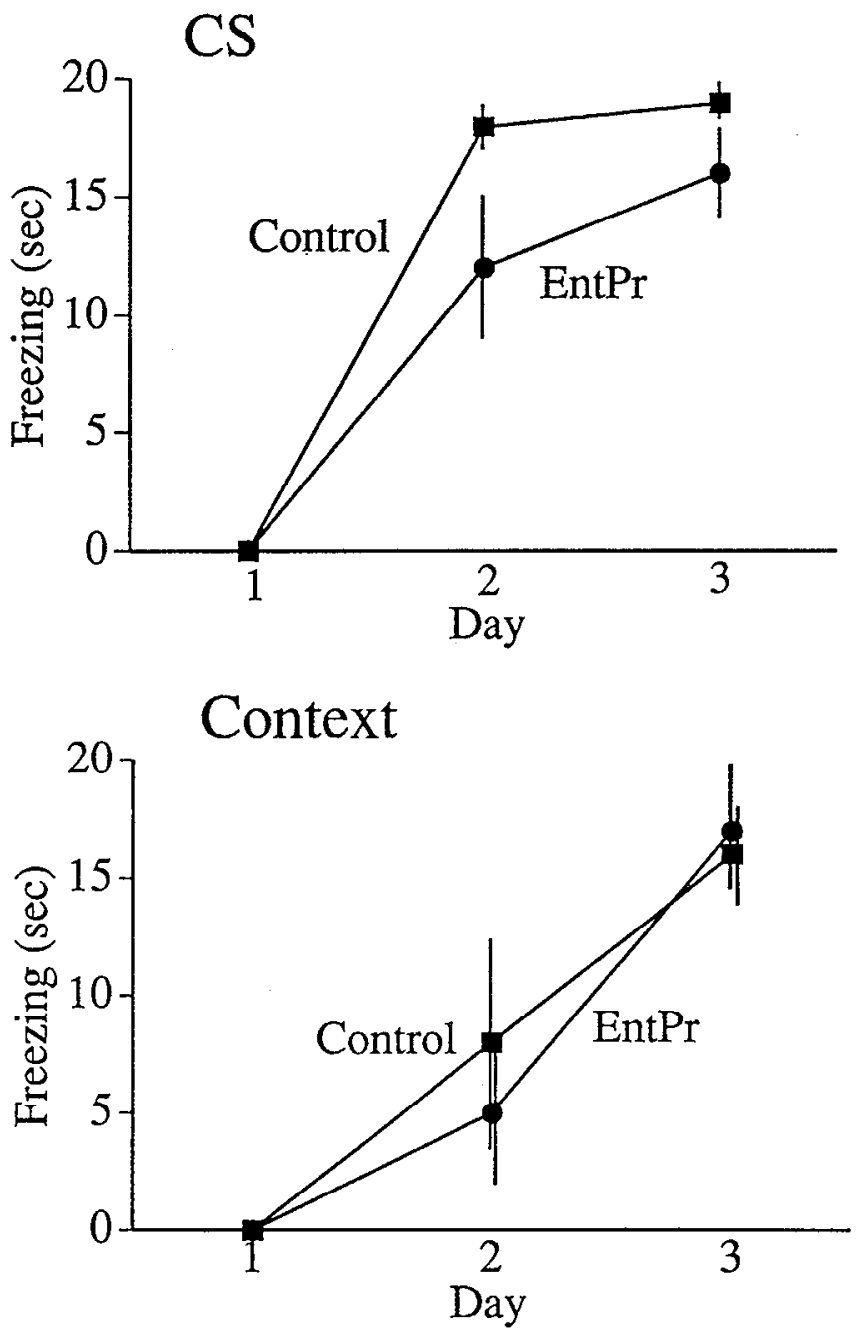

Figure 4. Effects of lesions of the combined lesion of the entorhinal and perirhinal cortex (EntPr) on the acquisition of conditioned freezing to the conditioned stimulus ( $C S$; a tone) and to the background context. Error bars represent $\mathrm{SE}$ above and below the mean (EntPr, $n=5$; control, $n=8$ ).

to which the animals were responding (see Fig. 6). Evaluation of the data with post hoc $t$ tests revealed that fornix lesions affect contextual conditioning on day $2[t(26)=3.08, p<0.002]$ and day $3[t(26)=4.43, p<0.002]$.

\section{Discussion}

Recent studies have implicated the hippocampal formation in contextual fear conditioning (Selden et al., 1991; Kim and Fanselow, 1992; Phillips and LeDoux, 1992, 1994). In the present study, we attempted to determine how information from the environment might reach the hippocampus in the process of forming contextual representations during fear conditioning. We began by making lesions in the entorhinal cortex, which is believed to be an important link between neocortical sensory processing systems and the hippocampus (van Hoesen, 1982; Amaral, 1987; Amaral and Witter, 1989). As these lesions did not interfere with conditioning to either the context or an explicit CS, combined lesions of the entorhinal and perirhinal cortex were made. The perirhinal cortex is known to have a direct connection with the hippocampal formation (Kosel et al., 1983) and the entorhinal cortex may have been bypassed. Perirhinal cortex also has con- 


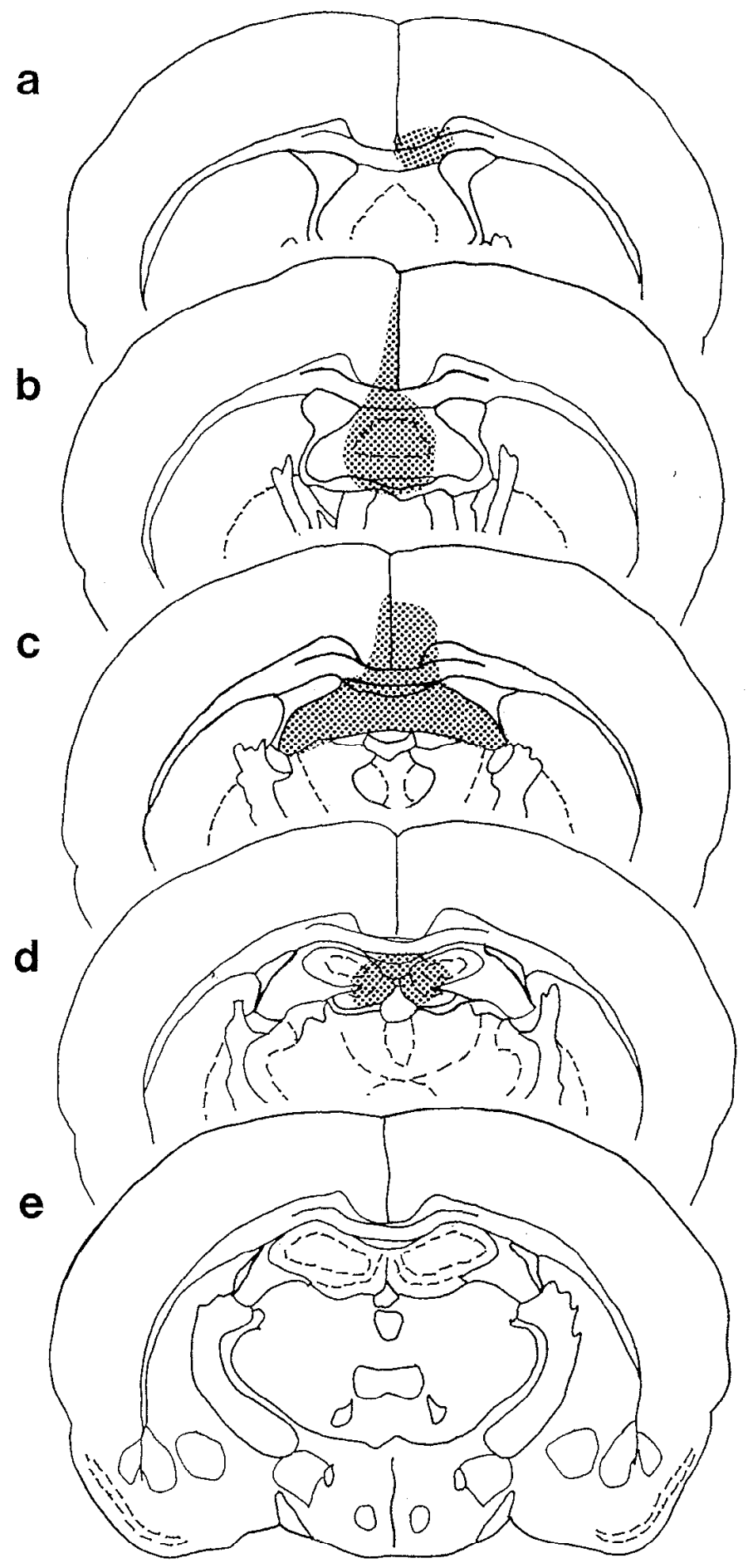

Figure 5. Serial frontal sections illustrating a typical lesion of the fornix $(F x)$. Sections are from rostral to caudal $(a-e)$, with stippling indicating the lesion site.

nections with the amygdala (Burwell and Amaral, 1993; Romanski and LeDoux, 1993), which is also required for contextual conditioning (Phillips and LeDoux, 1992). The combined EntPr lesions had no effect. Another major source of input and output connections of the hippocampus is the fornix (Swanson and Cowan, 1977; Amaral, 1987). In contrast to the Ent and EntPr lesions, fornix lesions interfered with conditioning to context but
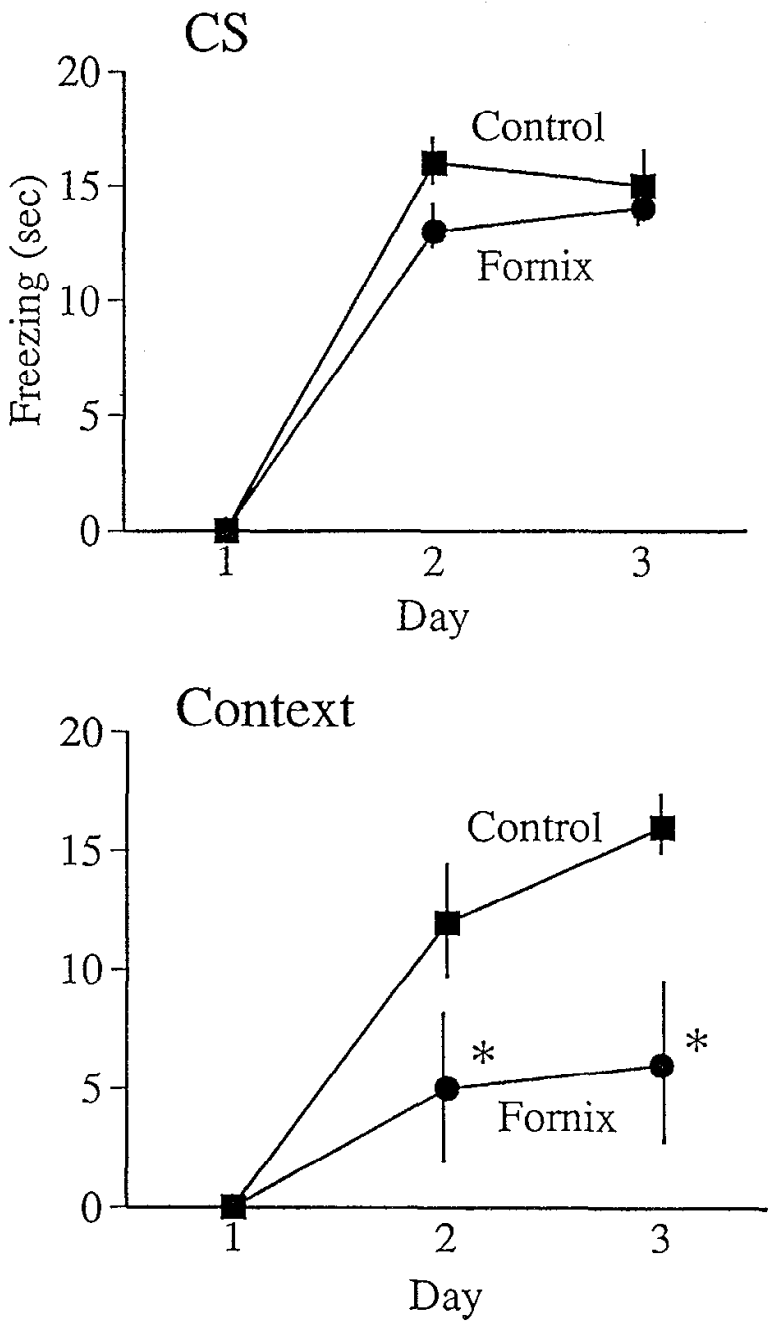

Figure 6. Effects of lesions of the fornix $(F x)$ on the acquisition of conditioned freezing to the conditioned stimulus ( $C S$; a tone) and to the background contcxt. Error bars represent SE above and below the mean; *, $p<0.01$ (Fx, $n=8$; control, $n=6$ ).

not to an explicit CS. Since the fornix fibers link the hippocampus with subcortical structures, these results suggest that the conditioning of fear reactions to background or contextual stimuli may depend more on the transmission of information between subcortical areas and the hippocampus.

Rhinal cortex lesions (including lesions of the entorhinal, perirhinal, or parahippocampal regions) disrupt performance of a variety of hippocampal-dependent tasks (Olton et al., 1978; Staubli et al., 1986; Murray et al., 1989; Zola-Morgan et al., 1989; Meunier et al., 1990; Otto et al., 1991; Bunsey and Eichenbaum, 1993; Mumby and Pinel, 1994; Nagahara et al., 1995). Furthermore, recent studies suggest that lesions of this area are more effective than, or at least as effective as lesions of the hippocampus in producing amnestic effects on delayednonmatch-to-sample, or delayed-match-to-sample tasks, in both rats and monkeys (Zola-Morgan and Squire, 1985; Gaffan and Murray, 1992; Mumby and Pinel, 1994). Excitotoxic lesions of entorhinal cortex impair retention on tasks learned 2-3 weeks prior to surgery, but leave animals relatively unimpaired on tasks learned 4-6 weeks prior to surgery (Cho et al., 1993). Also, infusion of CNQX, AP5, or muscimol into the entorhinal cortex impairs performance on passive avoidance tasks (Ferreira et al., 


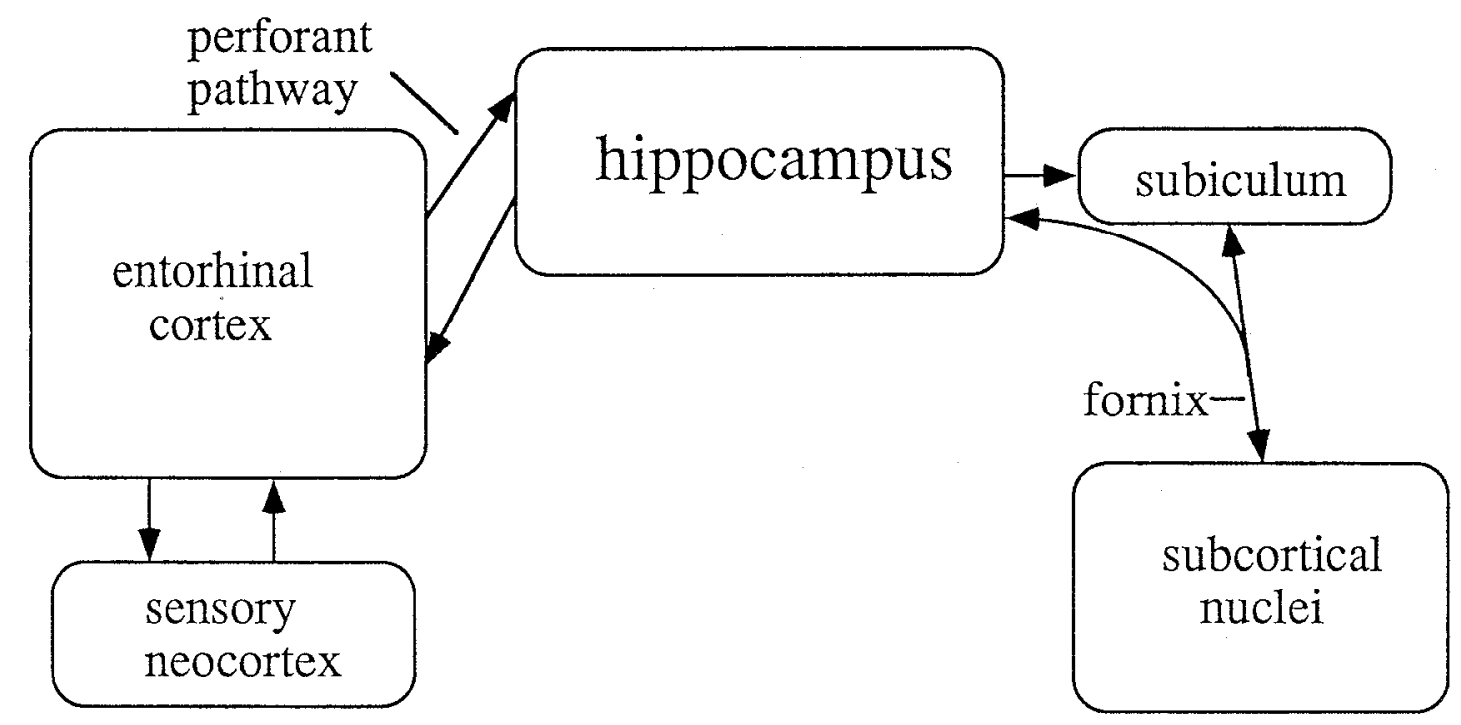

Figure 7. Schematic of the circuit for neocortical and subcortical hippocampal input and output.

1992; Izquierdo et al., 1993), which also depend upon the hippocampus (O'Keefe and Nadel, 1978; Gray, 1982). In spite of the fact that entorhinal cortex lesions typically replicate the effects of hippocampal lesions, entorhinal cortex lesions failed to affect contextual fear conditioning in the present study. A similar result (no effect of Ent lesions) has been recently reported in abstract form for two other hippocampal-dependent tasks (Jarrard and Hyko, 1994; Otto and Schiller, 1994).

Fornix lesions, which are often treated as analogous to lesions of the hippocampus (e.g., Gaffan, 1974; Eichenbaum et al., 1986), interfere with performance on spatial as well as nonspatial tasks (e.g., Eichenbaum et al., 1988; Raffaele and Olton, 1988). The present results are consistent with the relevant literature using lesions of the fornix as a method for evaluating the role of the hippocampal memory system, as fonix lesiuns disrupted contextual fear conditioning. Thus, subcortical inputs and outputs of the hippocampus (or cortical inputs that bypass the entorhinal and perirhinal cortex) may be sufficient in providing information to the hippocampus for conditioning to context and in controlling the transmission of information to others areas, such as the amygdala, which are required for the acquisition of the aversive association and the control of conditioned responses (Davis, 1992; Kapp, 1990; LeDoux, 1992, 1995). These findings raise questions concerning the exact inputs to the hippocampus necessary for conditioning to context (see Fig. 7).

Although entorhinal cortex lesions did not disrupt contextual fear conditioning, they affected passive avoidance conditioning. This is of interest given that passive avoidance is often considered a form of contextual fear conditioning, and like contextual fear conditioning is dependent on the hippocampus (O'Keefe and Nadel, 1978; Gray, 1982; Selden el al., 1991). However, passive avoidance is most similar to the form of contextual conditioning that involves the delivery of the US without a CS. We have termed this "foreground contextual conditioning," to distinguish it from the situation in which an explicit CS, such as a tone, is paired with a US, and the contextual stimuli are in the background against this primary association (Phillips and LeDoux, 1994). Given that we found that hippocampal lesions affected background but not foreground contextual conditioning (Phillips and LeDoux, 1994), it is surprising that entorhinal cortex lesions have an effect on passive avoidance conditioning (a foreground contextual task) but have no effect on background contextual conditioning. But the role of the hippocampus in passive avoidance and background contextual fear conditioning may be different. That this is the case is suggested by the fact that neither the Ent or control animals exhibited much freezing behavior in the passive avoidance chamber. Although contextual conditioning and passive avoidance learning are superficially similar, they may be very different processes. It may be that the entorhinal cortex is required, not as an input to the hippocampus in passive avoidance conditioning, but as an output required to control instrumental behavior. Except for the presence of the "safe" platform in the passive avoidance task, the chambers were similar in the two tasks. However, the presence of the safe area makes the shock avoidable and drastically alters the behavioral opportunities available to the animal. While classically conditioned freezing is an involuntarily elicited respondent controlled by antecedents (Bouton and Bolles, 1980), the passive avoidance response is a voluntary instrumental response, an operant controlled by its consequences. As a result, passive avoidance may require cortical systems and their interactions with basal ganglia motor systems (Mink and Thach, 1993). Thus, lesions of the entorhinal cortex might have been effective at disrupting acquisition of the passive avoidance task because contextual information from the hippocampus cannot reach these motor circuits rather than because sensory information cannot reach the hippocampus. Resolution of the difference between the effects of contextual fear conditioning and passive avoidance conditioning might suggest some important insights into the anatomical systems that allow the hippocampus to control fundamentally different kinds of behaviors, perhaps as fundamentally different as voluntary versus involuntary emotional responses, in reaction to the same stimulus information.

Although it is widely believed that the entorhinal cortex is a critical relay for sensory inputs to the hippocampus, recent studies have suggested that some sensory (visual) information reaches the hippocampus directly from the thalamus (Mizumori et al., 1993) and visual association cortex (Shi et al., 1994). Whether these pathways or other more indirect routes might be involved is not known. Also, it is unclear whether the entorhinal cortex might normally be the preferred pathway and the subcortical routes are only used when the preferred pathway is damaged. 
What is clear, though, is that in the absence of the entorhinal cortex, contextual conditioning can proceed at a normal rate. Further study is required to determine the exact afferent pathways to the hippocampus that are necessary for background or contextual fear conditioning.

\section{References}

Aggleton JP (1992) The amygdala: neurobiological aspects of emotion, memory, and mental dysfunction. New York: Wiley-Liss.

Amaral DG (1987) Memory: anatomical organization of candidate brain regions. In: Handbook of physiology, Sec 1, Neurophysiology, Vol 5, Higher functions of the brain (Plum F, ed), pp 211-294. Bethesda, MD: American Physiological Society.

Amaral DG, Witter MP (1989) The three-dimensional organization of the hippocampal formation: a review of anatomical data. Neuroscience 31:571-591.

Blanchard RJ, Blanchard DC (1969) Crouching as an index of fear. J Comp Physiol Psychol 67:370-375.

Bouton ME, Bolles RC (1980) Conditioned fear assessed by freezing and by the suppression of three different baselines. Anim Learn Behav 8:429-434.

Bunsey M, Eichenbaum $\mathbf{H}$ (1993) Critical role of the parahippocampal region for paired-associate learning in rats. Behav Neurosci 107:740747.

Burwell RD, Amaral DG (1993) A PHA-L study of projections from perirhinal cortex in the rat. Soc Neurosci Abstr 23:352.

Cho YH, Beracochea D, Jaffard R (1993) Extended temporal gradient for the retrograde and anterograde amnesia produced by ibotneate entorhinal cortex lesions in mice. J Neurosci 13:1759-1766.

Davis M (1992) The role of the amygdala in conditioned fear. In: The amygdala: neurobiological aspects of emotion, memory, and mental dysfunction (Aggleton JP, ed), pp 255-306. New York: Wiley-Liss.

Deacon TW, Eichenbaum H, Rosenberg P, Eckmann K (1983) Afferent connections of the perirhinal cortex in the rat. J Comp Neurol 220: $168-190$.

Edeline J-M, Dutrieux G, Nuenschwander-El Massioui N (1990) Multiunit changes in hippocampus and medial geniculate body in freebehaving rats during acquisition and retention of a conditioned response to a tone. Behav Neural Biol 50:61-79.

Eichenbaum H, Fagan A, Cohen NJ (1986) Normal olfactory discrimination learning set and facilitation of reversal learning after combined and separate lesions of the fornix and amygdala in rats: implications for preserved learning in amnesia. J Neurosci 6:1876-1884.

Eichenbaum H, Fagan A, Mathews P, Cohen NJ (1988) Hippocampal system dysfunction and odor discrimination learning in rats: impairment or facilitation depending on representational demands. Behav Neurosci 102:3531-3542.

Fanselow MS (1980) Conditional and unconditional components of postshock freezing. Pavlovian J Biol Sci 15:177-182.

Ferreira MBC, Da Silva RC, Medina JH, Izquierdo I (1992) Late posttraining memory processing by entorhinal cortex: involvement of NMDA and GABAergic receptors. Pharmacol Biochem Behav 41: $767-771$.

Gaffan D (1974) Recognition impaired and association intact in the memory of monkeys after transection of the fornix. J Comp Physiol Psychol 86:1100-1109.

Gaffan D, Murray EA (1992) Monkeys (Macaca fascicularis) with rhinal cortex ablations succeed in object discrimination learning despite 24-hr intertrial intervals and fail at matching to sample despite double sample presentations. Behav Neurosci 106:30-38.

Gray J (1982) The neuropsychology of anxiety. London: Oxford UP.

Izquierdo I, Da Silva RC, Bueno e Silva M, Quillfeldt JA, Medina JH (1993) Memory expression of habituation and of inhibitory avoidance is blocked by CNQX infused into the entorhinal cortex. Behav Neural Biol 60:5-8.

Kapp BS, Wilson A, Pascoe IP, Supple W, Whalen PI (1990) A neuroanatomical systems analysis of conditioned bradycardia in the rabbit. In: Learning and computational neuroscience: foundations of adaptive networks (Gabricl M, Moore J, eds), pp 53-90. Cambridge, MA; MIT Press.

Kapp BS, Whalen PJ, Supple WF, Pascoe JP (1992) Amygdaloid contributions to conditioned arousal and sensory information processing. In: The amygdala: neurobiological aspects of emotion, memory, and mental dysfunction (Aggleton JP, ed), pp 229-254. New York: WileyLiss.

Kim JJ, Fanselow MS (1992) Modality-specific retrograde amnesia of fear. Science 256:675-677.

Kosel KC, van Hoesen GW, Rosene DL (1983) A direct projection from the perirhinal cortex (area 35 ) to the subiculum in the rat. Brain Res 269:437-351.

Krettek JE, Price JL (1977) Projections from the amygdaloid complex to the cerebral cortex and thalamus in the rat and cat. J Comp Neurol 172:687-722.

LeDoux JE (1992) Brain mechanisms of emotion and emotional learning. Curr Opinion Neurobiol 2:191-198.

LeDoux JE (1995) Emotion: clues from the brain. Annu Rev Psychol 46:209-235.

LeDoux JE, Sakaguchi A, Reis DJ (1984) Subcortical efferent projections of the medial geniculate nucleus mediate emotional responses conditioned by acoustic stimuli. J Neurosci 4:683-698.

McDonald AJ, Jackson TR (1987) Amygdaloid connections with posterior insular and temporal cortical areas in the rat. J Comp Neurol 262:59-77.

Meunier M, Murray EA, Bachevalier J, Mishkin M (1990) Effects of perirhinal cortical lesions on visual recognition memory in rhesus monkeys. Soc Neurosci Abstr 16:616.

Mink JW, Thach WT (1993) Basal ganglia intrinsic circuits and their role in behavior. Curr Opinion Neurobiol 3:950-957.

Mizumori SJY, McNaughton BL, Barnes CA, Fox KB (1989) Preserved spatial coding in hippocampal CAl pyramidal cells during reversible suppression of CA3c output: evidence for pattern completion in hippocampus. J Neurosci 9:3915-3928.

Mumby DG, Pinel JPJ (1994) Rhinal cortex lesion and object recognition in rats. Behav Neurosci 108:11-18.

Murray EA, Bachevalier J, Mishkin M (1989) Effects of rhinal cortical lesions on visual recognition memory in rhesus monkeys. Soc Neurosci Abstr 15:342.

Nagahara AH, Otto T, Gallagher M (1995) Entorhinal-perirhinal lesions impair performance on two versions of place learning in the Morris water maze. Behav Neurosci 109:3-9.

O'Keefe J, Nadel L (1978) The hippocampus as a cognitive map. London: Oxford UP.

Olton DS, Walker JA, Gage FH (1978) Hippocampal connections and spatial discrimination. Brain Res 139:295-308.

Otto TA, Schiller KM (1994) Effects of entorhinal cortex aspirations on odor- and context-guided fear conditioning. Soc Neurosci Abstr 413.19 .

Otto T, Schottler F, Staubli U, Eichenbaum H, Lynch G (1991) Hippocampus and olfactory discrimination learning: effects of entorhinal cortex lesions on olfactory learning and memory in a successive-cue, go-no-go task. Behav Neurosci 105:11-119.

Paxinos G, Watson C (1986) The rat brain in stereotaxic coordinates. Sydney: Academic.

Phillips RG, LeDoux JE (1992) Differential contribution of amygdala and hippocampus to cued and contextual fear conditioning. Behav Neurosci 106:274-285.

Phillips RG, LeDoux JE (1994) Lesions of the dorsal hippocampal formation interfere with background but not foreground contextual fear conditioning. Learn Memory 1:34-44.

Raffaele KC, Olton DS (1988) Hippocampal and amygdaloid involvement in working memory for nonspatial stimuli. Behav Neurosci 102: 349-355.

Romanski LM,LeDoux JE (1993) Information cascade from primary auditory cortex to the amygdala: corticocortical and corticoamygdaloid projections of temporal cortex in the rat. Cereb Cortex 3:515532.

Swanson LW, Cowan WM (1977) An autoradiographic study of the organization of the efferent connections of the hippocampal formation in the rat. J Comp Neurol 172:49-84.

Selden NRW, Everitt BJ, Jarrard LE, Robbins TW (1991) Complimentary roles for the amygdala and hippocampus in aversive conditioning to explicit and contextual cues. Neuroscience 42:335-350.

Staubli U, Fraser D, Kessler M, Lynch G (1986) Studies on retrograde and anterograde amnesia of olfactory memory after denervation of the hippocampus by entorhinal cortex lesions. Behav Neural Biol 46: 432-444.

Swanson LW, Kohler C (1986) Anatomical evidence for direct projec- 
tions from the entorhinal area to the entire cortical mantle in the rat. J Neurosci 6:3010-3023.

Turner BH, Zimmer J (1984) The architecture and some of the interconnections of the rat's amygdala and lateral periallocortex. J Comp Neurol 227:540-557.

van Hoesen GW, Pandya DN, Butters N (1975) Some connections of the entorhinal (area 28) and perirhinal (area 35) cortices of the rhesus monkey. Brain Res 95:1-24
Zola-Morgan S, Squire LR (1985) Medial temporal lesions in monkeys impair memory on a variety of lasks semsitive to human amnesia. Behav Neurosci 99:22-34.

Zola-Morgan S, Squire LR, Amaral DG, Suzuki WA (1989) Lesions of the perirhinal and parahippocampal cortex that spare the amygdala and hippocampal formation produce severe memory impairment. $\mathrm{J}$ Neurosci 9:4355-4370. 